Rakenteiden Mekaniikka (Journal of Structural Mechanics)

Vol. 50, No 3, 2017, pp. 216 - 219

https://rakenteidenmekaniikka.journal.fi/index

https://doi.org/10.23998/rm. 64643

(c) The author(s) 2017.

Open access under CC BY-SA 4.0 license.

\title{
Numerical modelling of rock materials with polygonal finite elements
}

\author{
Timo Saksala
}

Summary. This article presents some preliminary results on numerical modeling of rock materials with polygonal finite elements. A method to describe the rock microstructure based on Voronoi diagrams, representing the rock grain texture, is sketched. In this method, the minerals constituting the rock are represented as Voronoi cells which themselves are polygonal finite elements. A three-point bending problem under plane stress linear elasticity condition is solved in order to compare the performance of polygonal elements to ordinary finite elements. Moreover, it is demonstrated by solving the stress state in uni-axial compression that the heterogeneity described with the present method results in short-range tensile stresses which could initiate mode-I cracks.

Key words: polygonal elements, Voronoi tessellation, rock microstructure, FEM

Received 9 June 2017. Accepted 10 August 2017. Published online 21 August 2017

\section{Introduction}

Polygonal finite elements have been drawing increasing attention during the last 15 years. In comparison to the standard finite elements, these elements offer greater flexibility in meshing arbitrary geometries, better accuracy in the numerical solution, better description of certain materials, and less locking-prone behavior under volume-preserving deformation [1]. With these elements, some numerical problems, such as checkerboarding in topology optimization, can be alleviated [2]. Polygonal discrete elements based on Voronoi diagrams is already an established method in geomechanics (see, for example, UDEC manual [3]). On the other hand, the disadvantages of the polygonal finite element method include less sparse system matrix and the need for a higher order numerical integration quadrature to achieve high-accuracy.

In the present paper, some preliminary results on a project of modelling rock materials with polygonal finite elements based on the Voronoi tessellation are presented. Namely, a method to describe the rock microstructure based on the Voronoi tessellation is sketched. At this preliminary stage of developments, only 2D linear elasticity problems are considered as numerical examples. 

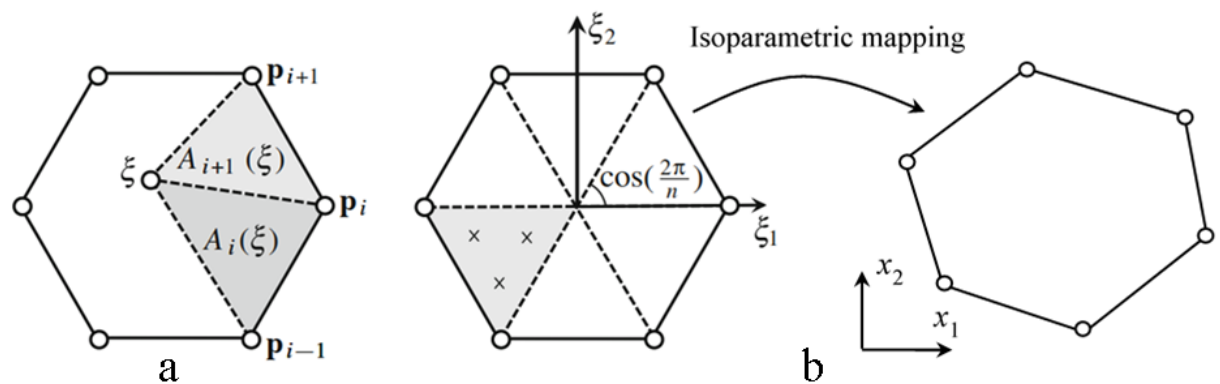

Figure 1. Illustration of the triangular areas used in the definition of Wachspress shape function (a), and the triangulation of the reference regular polygon, integration points of each triangle, and the isoparametric mapping to a physical element (b).

\section{Polygonal finite elements}

The standard isoparametric mapping from a reference element to the physical element can be used with the polygonal finite elements as well [1], as illustrated in Figure 1b. Here, the work by Talischi et al. [2] based on the Wachspress rational interpolation functions, providing $C^{0}$-continuity, is followed. For a reference $n$-gon (see Figure $1 \mathrm{~b}$ for hexagon), the Wachspress shape function at node $i$ is

$$
N_{i}(\boldsymbol{\xi})=\frac{\alpha_{i}(\boldsymbol{\xi})}{\sum_{j=1}^{n} \alpha_{j}(\boldsymbol{\xi})}, \quad \alpha_{i}(\boldsymbol{\xi})=\frac{A\left(\mathbf{p}_{i-1}, \mathbf{p}_{i}, \mathbf{p}_{i+1}\right)}{A\left(\mathbf{p}_{i-1}, \mathbf{p}_{i}, \boldsymbol{\xi}\right) A\left(\mathbf{p}_{i}, \mathbf{p}_{i+1}, \boldsymbol{\xi}\right)}
$$

where $A(a, b, c)$ denotes the signed area of triangle $a, b, c$ (see see Figure 1a). The numerical integration is carried out by sub-dividing the reference polygon into triangles and using a three integration points scheme for each triangle (resulting $3 n$ integration points for each $n$-gon), as illustrated in Figure 1b. The variational (weak) formulation of the polygonal finite elements is fully standard, see [1] for details.

\section{Rock microstructure description}

Polycrystalline materials such as rocks can be naturally described with non-regular meshes of polygonal elements. Here, the minerals constituting the rock are represented by random clusters of Voronoi cells which themselves are polygonal finite elements. For this end, the PolyMesher Matlab code by Talischi et al. [4] is employed. This code generates 2D Voronoi diagrams (tessellations) consisting of centroidal Voronoi cells, which are then used as polygonal finite element meshes in the present work. The rock material is assumed to consist of tree main minerals (quartz, feldspar, biotite) which have their respective material properties. Thereby, the rock heterogeneity can be described. This method is illustrated in Figure 3a and b where two different realizations of the numerical rock sample with 2000 grains (Voronoi cells/polygon elements) are generated. In the first sample, the generating seeds are random yielding a realistic rock microstructure while in the second the polygons in the mesh are centroidal, i.e. the generating seeds are at the centroids of the polygons (CVT mesh), leading to a more regular cell distribution.

\section{Numerical examples}

The three-point bending problem of a linear elastic (plane-stress) beam is solved first in order to compare the performance of the polygonal elements to that of standard CST 
(constant strain triangle) and bilinear rectangle elements. The material parameters are $E=200 \mathrm{GPa}, \nu=0.3$, while the beam dimensions are $L=0.6 \mathrm{~m}, h=0.1 \mathrm{~m}$, and thickness $t=0.01 \mathrm{~m}$. The load is $1000 \mathrm{~N}$. The results, exploiting the symmetry of the problem, are shown in Figure 2. The results for maximum deflection at the mid-span of the bottom edge are normalized with the engineering beam theory solution of $u_{\text {beam }}=5.4 \times 10^{-5} \mathrm{~m}$.

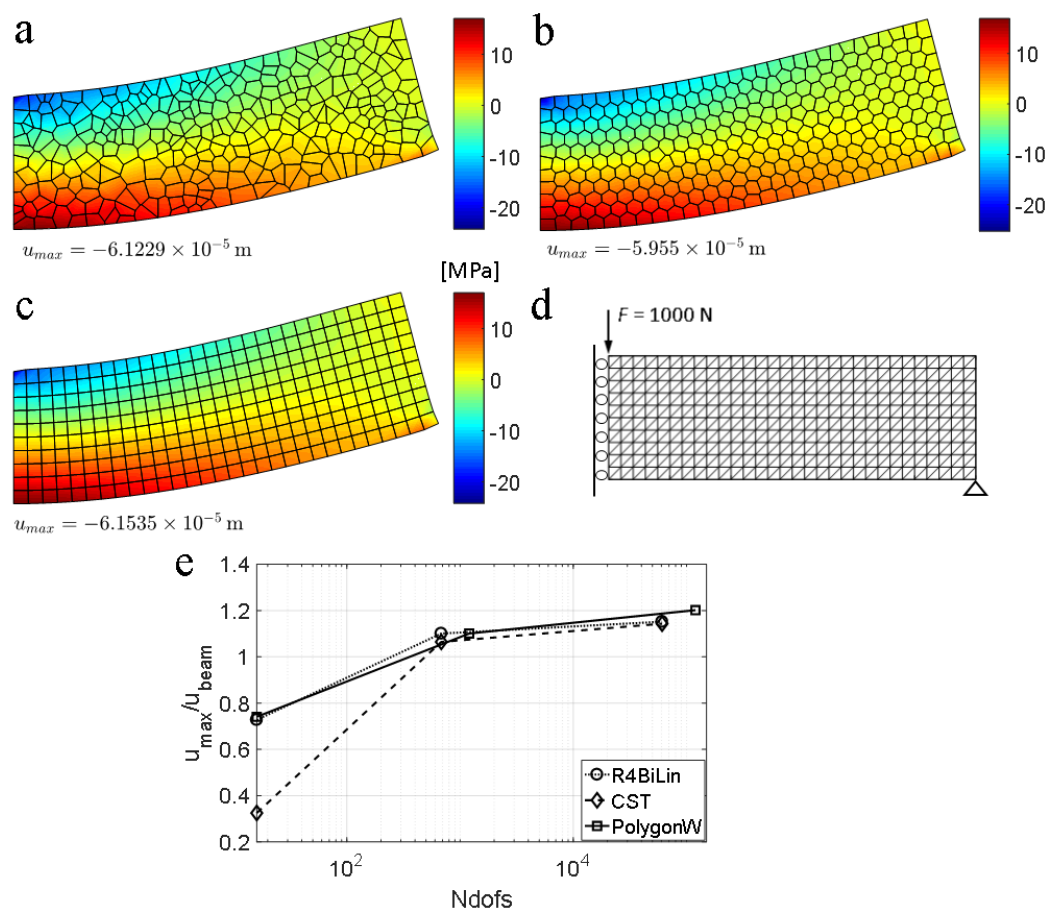

Figure 2. Results for 3-point bending of beam: examples of stresses and deformed meshes (1000-fold magnification) with 300 polygons with random seeds (a), with CVT mesh (b), and regular 4-gon mesh (c), a regular CST mesh with the boundary conditions (d), and the maximum normalized deflection as a function of the number DOFs for tested elements (e).

Figure 2a-c show the results for three different mesh structurations with 300 polygons. It should be noted that the result in Figure 2c is for polygonal mesh with Wachspress basis functions which are not identical to the 4-node bi-linear basis functions. Figure 2e shows that the 4-node rectangle and the Wachspress polygonal element perform better than CST element with coarse meshes (as expected).

Finally, the stress fields in uniaxial compression of the numerical rock specimen in Figure $3 \mathrm{a}$ are solved. The loading is such that the nominal stress for a homogeneous specimen is $130 \mathrm{MPa}$. The mineral elastic properties are as follows: Quartz $(33 \%) E=$ $80 \mathrm{GPa}, \nu=0.17$; Feldspar $(50 \%) E=60 \mathrm{GPa}, \nu=0.29$; Biotite $(17 \%) E=20 \mathrm{GPa}$, $\nu=0.2$.

The results for the stress fields in both co-ordinate directions in Figure $3 \mathrm{~b}$ and $\mathrm{c}$ display the high heterogeneity of the numerical specimen. Moreover, the transverse stress (x-direction) at many locations exceeds $20 \mathrm{MPa}$ so that these short-range tensile stresses would generate mode-I cracks parallel to the loading direction.

\section{Conclusions}

Polygonal finite elements method was employed in this paper for numerical description of rock materials. The rock microstructure was modelled by Voronoi diagrams with the 


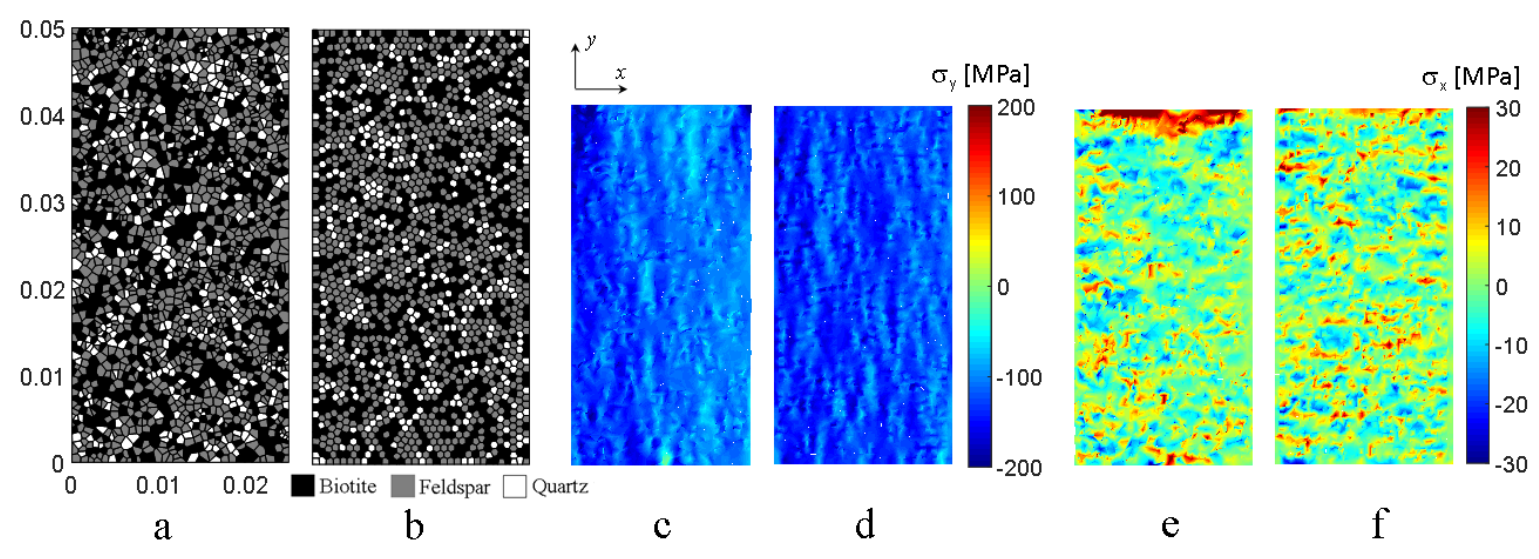

Figure 3. Numerical specimen with 2000 polygons with random seeds (specimen1) (a), with centroidal Voronoi tessellation (specimen2) (b), the stress distribution in $y$-direction under static compression of specimen1 (c), of specimen2 (d), and the stress distribution in $x$-direction under static compression of specimen1 (e), specimen2 (f).

constituting minerals being represented as random clusters of Voronoi cells (polygonal finite elements). The numerical tests demonstrated that the performance of even highly non-structured meshes is acceptable for present purposes. The uniaxial compression test under a nominal stress of $130 \mathrm{MPa}$ showed that the heterogeneity of the numerical specimen causes short-range tensile stresses that exceed the tensile strength of most rocks. Therefore, it can be concluded that the present approached has a good potential to be further developed to include a fracture model. This avenue will be pursued in the future studies of the approach.

\section{Acknowledgments}

This research was funded by Academy of Finland under grant number 298345.

\section{References}

[1] N. Sukumar, A. Tabarraei. Conforming polygonal finite elements. International Journal for Numerical Methods in Engineering, 61:2045-2066, 2004. https://doi.org/10.1002/nme. 1141

[2] C. Talischi, G.H. Paulino, A. Pereira, I.F.M. Menezes. PolyTop: a Matlab implementation of a general topology optimization framework using unstructured polygonal finite element meshes Structural and Multidisciplinary Optimization, 45:329-357, 2012. https://doi.org/ 10.1007/s00158-011-0706-z

[3] Itasca Consulting Group, Inc.: Universal Distinct Element Code: Theory and Background[R]. Itasca Consulting Group, Inc., Min- neapolis, 2013.

[4] C. Talischi, G.H. Paulino, A. Pereira, I.F.M. Menezes. PolyMesher: a general-purpose mesh generator for polygonal elements written in Matlab. Structural and Multidisciplinary Optimization, 45:309-328, 2012. https://doi.org/10.1007/s00158-011-0696-x

Timo Saksala

Laboratory of Civil Engineering

Tampere University of Technology

P.O.Box 600, FIN-33101, Tampere, Finland

timo.saksala@tut.fi 